\title{
Does Social Media Communication Style Influence Online Consumer Experience and Behavior?: An Abstract
}

\author{
Renato Hübner Barcelos, Danilo Correa Dantas, and Sylvain Senecal
}

\begin{abstract}
We investigate how the use of a human or a corporate tone of voice (ToV) on hotels' brand pages influences information search and customer attitudes on social media. Given that social media enables a closer contact with customers, brands have the option to present themselves in a more human or corporate way. We performed an online pilot study and an eye-tracking experiment in a lab setting to test the effects of a human or corporate tone of voice on social media. In the pilot study ( $n=105$ ), we show that participants on the more "human" page condition experienced increased closeness toward the hotel and more hedonic value in the experience with the page. As a result, their attitude toward the hotel was also more favorable. In the eye-tracking experiment $(n=30)$, we show that participants looking at the more "corporate" page had more fixations and increased cognitive load in concentrated areas such as the posts' texts and the hotel information in the page header. Participants looking at the more "human" page had more spread fixations along the page and increased cognitive load on the page header and other pictures. The findings of the studies suggest that the choice of a more human style of communication on the brand page increased customers' hedonic value, and, as a result, they engaged in a more heuristic pattern of information search, paying more attention to peripheral cues such as pictures. This work contributes to the understanding of the branding of hospitality services on social media, highlighting the relationship between the ToV chosen by the brand and customer's experiences and browsing behavior.
\end{abstract}

References Available Upon Request

\author{
R. H. Barcelos \\ UQAM, Montreal, Canada \\ e-mail: hubner_barcelos.renato@uqam.ca \\ D. C. Dantas · S. Senecal $(\bowtie)$ \\ HEC Montreal, Montreal, Canada \\ e-mail: danilo.dantas@hec.ca; ss@hec.ca
}

